\title{
Urbane Kinokultur
}

Das Lichtspieltheater in der Großstadt 1895-1949

Wolfgang Flügel, Merve Lühr, Winfried Müller (Hg.)

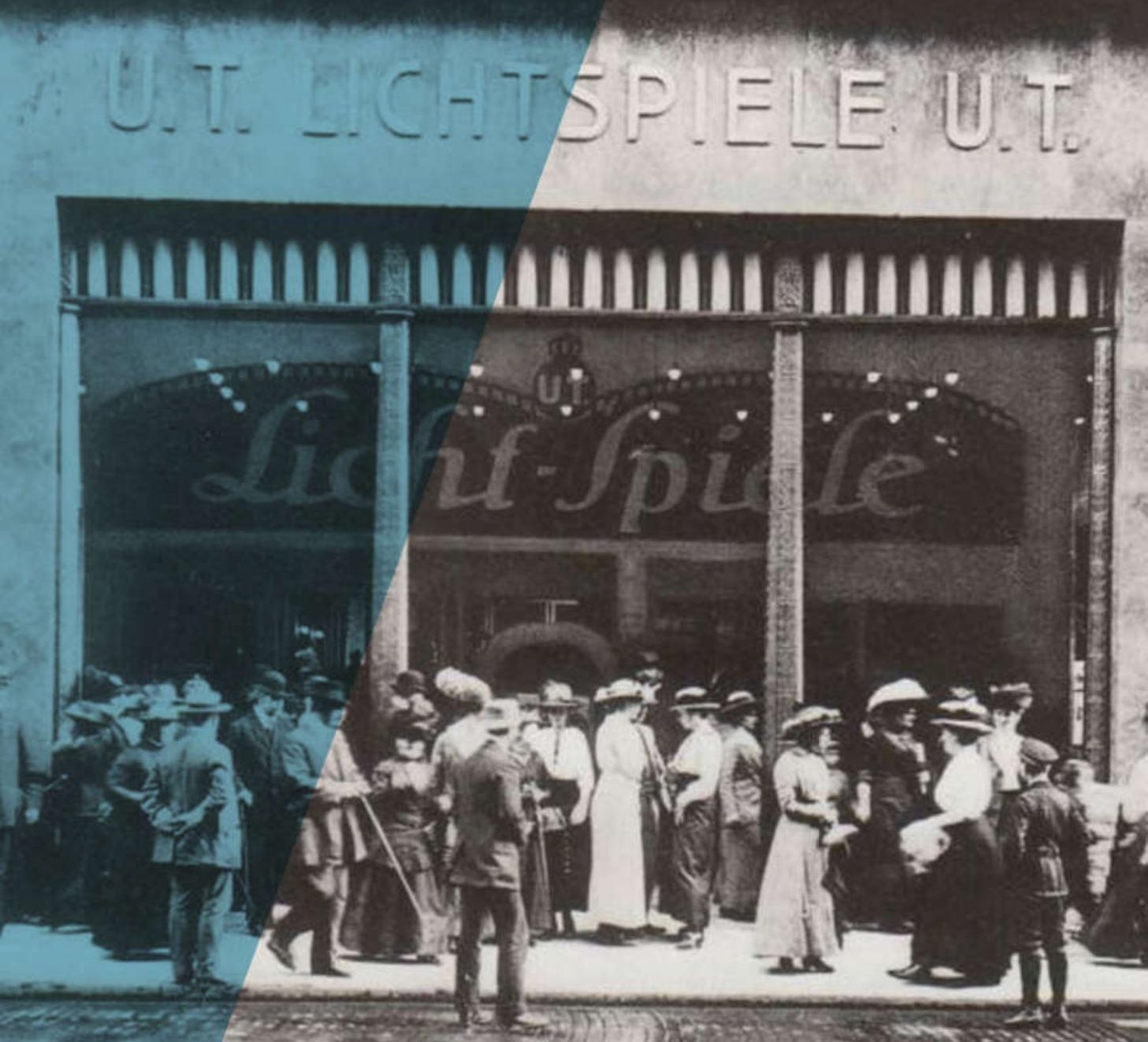





\section{Urbane Kinokultur \\ Das Lichtspieltheater in der Großstadt 1895-1949}

Wolfgang Flügel, Merve Lühr, Winfried Müller (Hg.)

in Zusammenarbeit mit Sophie Döring und Lennart Kranz 


\section{Impressum}

ISGV digital. Studien zur Landesgeschichte und Kulturanthropologie 2

herausgegeben von Enno Bünz, Andreas Rutz, Joachim Schneider und Ira Spieker

Redaktion:

Sophie Döring, Wolfgang Flügel, Merve Lühr, Winfried Müller, Susanne Müller

Layout: Josephine Rank, Berlin Technische Umsetzung (barrierefreies PDF): Klaas Posselt, einmanncombo Umschlaggestaltung: Josephine Rank nach einem Entwurf von Linda S. Gableske unter Verwendung einer Fotografie der U.T. Lichtspiele, Dresden, von 1913 (Quelle: https://filmtheater.square7.ch/ wiki/index.php?title=Datei:Dresden_UT_1913_ PK.jpg\#mw-navigation).

(C) Dresden 2020

Institut für Sächsische Geschichte und Volkskunde Zellescher Weg 17| 01069 Dresden

Bibliografische Information der Deutschen Nationalbibliothek: Die Deutsche Nationalbibliothek verzeichnet diese Publikation in der Deutschen Nationalbibliografie; detaillierte bibliografische Daten sind im Internet über http://dnb.ddb.de abrufbar.

Alle Rechte vorbehalten.

www.isgv.de

ISBN 978-3-948620-01-1

ISSN 2700-0613

DOI 10.25366/2020.41

Diese Maßnahme wird mitfinanziert durch

Steuermittel auf der Grundlage des vom

Sächsischen Landtag beschlossenen Haushaltes.

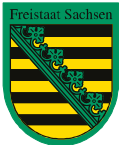




\section{Inhalt}

Wolfgang Flügel, Merve Lühr, Winfried Müller

Einleitung

Urbane Kinokultur: Das Beispiel Dresden

\section{Carola Zeh}

Bewegte Bilder - bewegte Geschichte

Zur Entwicklung des Kinos in Dresden

\section{Wolfgang Flügel}

Das frühe Dresdner Kino im Blick des Kinopioniers Heinrich Ott

\section{Sophie Döring}

Zwischen Kalklicht und Samtsessel

Mobile Kinopraxis in Sachsen 1896-1910

\section{Winfried Müller}

Ein neues Medium wird geadelt.

König Friedrich August III. von Sachsen geht ins Kino

\section{Mona Harring}

Kino- und Filmpolitik in Dresden zwischen 1945 und 1949 
Kino im urbanen Raum - Kino als urbaner Raum

\section{Lina Schröder}

Licht lockt Leute: Als der Mensch in die Schöpfung eingriff und Tag und Nacht aufhob - ein Werkstattbericht

\section{Kaspar Maase}

Kinderkino zwischen Kontrolle, Kommerz und Krawall

Anmerkungen zu einer Hamburger Initiative

aus dem frühen 20. Jahrhundert

\section{Fabian Brändle}

Wildwest und ein Schnäuzchen wie Clark Gable

Zürcher Kinokultur und Urbanität von 1900 bis 1940

\section{Sonja Neumann}

Konservenmusik und Elektrokapital

Tonfilmtechnik in München im Jahr 1929

\section{Sven Eggers}

Vor der Vorstellung

Die Herausbildung des Kinofoyers als urbane Gattung 183

\section{Merve Lühr}

Erstklassig und routiniert.

Das Lichtspieltheater als Arbeitsplatz 
Urbane Kinokultur: Die Klein- und Mittelstadt

\section{Niklas Hertwig}

„Film ab!" Max von Allweyer und seine Schulfilm-Unternehmung Lichtbildvorführungen an Volksschulen im ländlichen

Oberbayern 1926-1929

\section{Magdalena Abraham-Diefenbach}

Bellevue und Piast. Kino in den geteilten Städten

an der deutsch-polnischen Grenze 1945-1949

Jeanette Toussaint, Ralf Forster

Weltspiegel - Kino im 20. Jahrhundert.

Ein Ausstellungsprojekt

\section{Andrea Graf}

Publikum, Popcorn und Programm in der Provinz. Wie Kinokultur im ländlichen Raum funktioniert - 
Die Klein- und Mittelstadt

Die Klein- und Mittelstadt

(

Die Klein- und Mittelstadt

Die Klein- und Mittelstadt

Die Klein- und Mittelstadt

(

(n)

(n)

Die Kieln- und

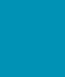$$
\text { . }
$$

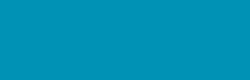$$
\text { ( }
$$
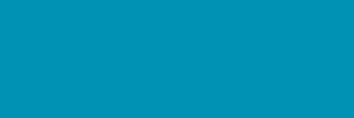


\title{
Publikum, Popcorn und Programm in der Provinz
}

\author{
Wie Kinokultur im ländlichen Raum funktioniert - \\ Ein Filmprojekt
}

Andrea Graf

\section{Einleitung: Kino in der Krise}

Aufgrund der Folgen der durch das Coronavirus SARS-CoV-2 ausgelösten Pandemie, die sich seit Januar 2020 von China aus ausbreitet, stehen auch die Kulturstätten weltweit unter einem enormen wirtschaftlichen Druck. In dem Bemühen, die Ausbreitung des Virus zu hemmen, wurden auch in Deutschland umfassende Maßnahmen, wie Ausgangsbeschränkungen, ergriffen und so sind Kinos genauso wie Theater und Museen seit dem 18. März 2020 geschlossen. Dies trifft den ländlichen Raum ebenso wie den städtischen. Um die Filmwirtschaft vor den Auswirkungen der „beispiellosen Bedrohung“" zu schützen, sind zeitnah Liquiditätsbeihilfen

$1 \quad$ Filmförderungsanstalt (FFA)-Presse- und Öffentlichkeitsarbeit: Corona-Krise. verabschiedet worden. Laut einer Pressemitteilung der Filmförderungsanstalt wurde ein gemeinsamer Hilfsfonds mit der Bundesbeauftragten für Kultur und Medien und den Länderförderern aufgelegt. In Zeiten von Ausgangssperren und Kontaktverboten greifen die Menschen zwangsläufig verstärkt auf Streamingdienste zurück. Doch auch abseits der von der CoronaPandemie ausgelösten Extremsituation gilt das Streaming als einer der Gründe, die die seit Jahren attestierte Krise des Kinos mit hervorgerufen haben. Dass das Kino als Kultur- und Freizeitort besonders im ländlichen Raum bedroht ist, ist unter anderem an den staatlich eingerichteten Fördermaßnahmen ablesbar. Seit Juli 2019 können sich Kinos auf eine finanzielle Förderung von bis zu 25.000 Euro Soforthilfe bewerben. Die Bundesregierung unterstützt dabei Modernisierungsmaßnahmen von Kinos in Gemeinden mit 
bis zu 25.000 Einwohnerinnen und Einwohnern, um die angespannte wirtschaftliche Situation für die Betreibenden zu verbessern. ${ }^{2}$

Anfang März 2020 folgt das Zukunftsprogramm Kino, dotiert mit 17 Millionen Euro, welches sich an Kinos in Städten mit bis zu 50.000 Einwohnerinnen und Einwohnern oder mit bis zu sieben Sälen richtet. ${ }^{3}$ Kulturstaatsministerin Monika Grütters (CDU) nennt das Kino in einem Zeitungsinterview einen soziokulturellen Ort, eine Begegnungsstätte oder auch eine geistige Tankstelle. ${ }^{4}$ Mithilfe der Förderprogramme soll nun ein jahrelanger Investitionsstau behoben werden, um etwa neue Kassensysteme, Projektoren und Sitze, eine modernere Heizungsanlage oder eine App zu bezahlen. Auch Fassade und Foyer können mit dem Geld aufgefrischt werden ${ }^{5}$. Einerseits begrüßt, wird das Zukunftsprogramm Kino vom Hauptverband der deutschen Filmtheater andererseits aufgrund der angelegten Förderkriterien kritisiert. Förderung solle, so wird die Vorstandsvorsitzende Christine Berg zitiert, noch stärker den Mittelstand in den Fokus stellen und inhabergeführten Traditionshäusern Anreize zur Modernisierung geben - jenseits von deren Standort und Programm, teilte der Verband mit. Die bisher vorgesehene Begrenzung auf kulturell besonders wertvolle Spielstätten ist nicht zielführend. ${ }^{6}$

Denn Kinos in größeren Städten können sich nur bewerben, sofern sie für ihr Programm

2 Presse- und Informationsamt der Bundesregierung (BPA): Pressemitteilung 227.

3 FFA Filmförderungsanstalt: Zukunftsprogramm Kino.

4 Alle kursiv gesetzten Formulierungen zitiert aus: Gohlisch: Grütters.

5 Ntv, Politik: Hilfsprogramm.

6 Ntv, Politik: Hilfsprogramm. ausgezeichnet wurden. Eine gezielte Förderung der Kinos in der Peripherie wie durch die Maßnahmen in den Jahren 2019 und 2020 begonnen, scheint demnach in der Branche selbst auf Unverständnis zu stoßen. ${ }^{7}$ Das Kino stellt in Dörfern und Städten außerhalb der Ballungsgebiete oftmals einen der wenigen Kulturorte dar. Für dessen Erhalt argumentiert Monika Grütters durchaus über seine Funktion in der Prävention gegen den zunehmenden Populismus und die Etablierung rechter Strukturen. ${ }^{8}$ Das Kino sei ein Ort der Teilhabe, der einen Blick über den eigenen Tellerrand und Diskussion mit Anderen ermögliche.

Und genau hier knüpft das Projekt zur Kinokultur im ländlichen Raum des Landschaftsverbands Rheinland - Institut für Landeskunde und Regionalgeschichte (LVR-ILR) mit seinen Fragen an: Zeichnen sich die Lichtspielhäuser abseits der Metropolen durch bestimmte Eigenschaften aus? Welche Funktionen erfüllen Kinos, besonders im ländlichen Raum? Konnte sich hier eine eigene Kinokultur entwickeln und erhalten? Diese Fragen möchten wir in filmischen Kinoporträts aus dem Rheinland thematisieren. In verschiedenen rheinischen Orten werden dazu seit 2019 in gegenwärtig existierenden Kinos Filmaufnahmen gemacht. In Interviews erzählen Betreiberinnen und Betreiber sowie Besucherinnen und Besucher ihre Kinogeschichte(n) und zeigen damit die Diversität von Motiven und Strategien von Kinomacherinnen und -machern abseits der rheinischen Großstädte auf. Bisher entstanden sind Kurzfilme über das Kur-Theater

7 Mensch: Zukunftsprogramm Kino; ntv, Politik: Hilfsprogramm.

8 Ntv, Politik: Hilfsprogramm. 
Hennef sowie das Corso Film Casino Kaldenkirchen. Geplant sind Forschungs- und Dreharbeiten in mindestens drei weiteren rheinischen Orten. Die Kinos in den unterschiedlichen Städten dienen als Ausgangspunkte für einen Zugang zum Thema, der oftmals ein biografischer ist.

Dabei betrachten wir das Ländliche und das Urbane nicht als Dichotomie, zumal diese Zuschreibungen für das Ballungsgebiet Rheinland nicht zutreffend sein können, sondern definieren ländlichen Raum und damit unser Forschungsgebiet als Peripherie, eben abseits der Metropolen: als Vorstädte, Dörfer, Klein- und Mittelstädte.

\section{Entstehungskontext des Projektes}

Das Projekt zur Kinokultur im ländlichen Raum entwickelte sich im Rahmen einer Kooperation zwischen dem LVR-ILR und den Filmemachern von Benda Film. Der aktuellen Kooperation ging eine erste Reihe von Kinoporträts im Ruhrgebiet voraus, die unter anderem vom Landschaftsverband Westfalen-Lippe gefördert wurde. Der Journalist und Filmemacher Daniel Huhn sowie der Kameramann Benjamin Leers beschäftigen sich mit weiteren Kollegen seit 2018 im Rahmen eines Dokumentarfilmprojekts mit dem Titel heimatkino.ruhr mit der städtischen Kinokultur im Ruhrgebiet. ${ }^{9}$ Ausgangspunkt war eine persönliche Erfahrung mit dem bereits geschlossenen Kino im Herkunftsort des Filmemachers Daniel Huhn: Bei jedem Besuch war dieser Ort für inn

9 https://heimatkino.de. aufgeladen mit den Erinnerungen und Emotionen der Filme, die dort gezeigt wurden und die er selbst als Jugendlicher dort gesehen hatte. ${ }^{10}$ So entstand die Idee, dem Kino, das sich seit über 100 Jahren dem Film widmet, selbst Filme zu widmen. ${ }^{11}$ Die Filmemacher begaben sich auf Recherche und entdeckten die Spuren einer einst vielfältigen Kinokultur zwischen Rhein und Ruhr. Die Zahlen aus den Kinohandbüchern belegten mehr als 500 Kinos zwischen Duisburg und Dortmund, heute sind es weniger als 50. Deren Standorte wurden auf einer Karte visualisiert. ${ }^{12}$ Über filmische Porträts ausgewählter Kinos sollte ein Blick geworfen werden auf die über 100-jährige Geschichte, aber vor allem auch auf die Gegenwart und Zukunft der städtischen Kinokultur.

In der ersten Staffel wurden dazu verschiedene Typen von Kinos ausgewählt, die im Laufe der letzten 70 Jahre entstanden waren. Diese Typologie entwickelten die Filmemacher von Benda Film aus ihrer Recherche der Kinolandschaft heraus. Von jedem dieser Typen existiert heute zumeist noch ein Kino. Überlebt haben die Häuser durch ihre unterschiedlichen Konzepte. Die entstandenen sieben Episoden von vier bis acht Minuten Länge über Kinos im Ruhrgebiet umfassen folgende Typen: das letzte klassische

10 Diese und die folgenden Informationen zur ersten Reihe des Filmprojekts sind sinngemäß dem Vortragsteil von Daniel Huhn (Benda Film) unseres gemeinsamen Beitrags auf der Tagung zur Urbanen Kinokultur entnommen.

11 Entstanden sind ein Dokumentarfilm "HeimatKino - Kinokultur im Ruhrgebiet", Produktion Benda Film 2018 (55 Min.), und sieben Episoden einer Webserie mit einzelnen Kinoporträts aus Essen, Gelsenkirchen, Dortmund, Duisburg und Bochum.

12 https://heimatkino.de/kinokarte. 
Bahnhofskino in Bochum, ein Autokino in Essen, ein kommunales Kino in Duisburg, ein Premierenkino in Essen, ein Nischenprogrammkino in Dortmund sowie ein Vorstadtkino in DortmundAplerbeck. ${ }^{13}$ Die Filmporträts geben einen Einblick in die unterschiedlichen Nutzungskonzepte und -strategien. Die meisten Kinos sind dabei, schon aufgrund ihrer Größe, eigentlich nur im urbanen Kontext denkbar.

Seit 2019 wird die Dokumentarfilmreihe nun um eine zweite Staffel erweitert, in welcher Kinoporträts über den ländlichen Raum des Rheinlands in Kooperation mit den Alltagskulturforscherinnen und -forschern des Landschaftsverbands Rheinland entstehen. ${ }^{14}$ Das LVR-ILR ist eine landeskundliche Forschungseinrichtung mit Sitz in Bonn und versteht sich als Kompetenzzentrum für Sprache, Geschichte und Alltagskultur des Rheinlandes. Als Ergebnis der Forschung entstehen neben Büchern, Ausstellungen und Vorträgen auch kulturanthropologische Filme. ${ }^{15}$ In unterschiedlichen Projekten wird zudem zum ländlichen Raum geforscht.

Über die Kurzfilme hinaus, in denen Konstanten und Transformationen des Kinos als Kulturort im ländlichen Raum des Rheinlands und dessen Bedeutung für Betreiberinnen und Betreiber sowie Besucherinnen und Besucher im Mittelpunkt stehen, wird empirisches Material erhoben, um die Funktion des Kinos für die jeweiligen Orte zu erfassen. Die entstehenden Kinoporträts haben wir mit einem Sammlungsaufruf nach Geschichten, Fotografien und Erinnerungen zur

13 https://heimatkino.de/episoden.

14 Zur Fortsetzung des Projektes konnten Mittel der regionalen Kulturförderung des LVR beantragt werden.

15 https://rheinische-landeskunde.lvr.de/de/volkskunde/filme/detailseite_24.html.
Kinokultur im Rheinland verknüpft. ${ }^{16}$ Zahlreiche Personen meldeten sich daraufhin bei uns, um beispielsweise auf ihr Lieblingskino aufmerksam zu machen. Diese Hinweise werden in die Auswahl der Drehorte für weitere Kinoporträts einfließen. Die im Rahmen des Projektes erhobenen Quellen sollen das Thema Freizeitkultur in unserem Archiv speisen und für eine Präsentation im Digitalen Portal Alltagskulturen aufbereitet werden. ${ }^{17}$

Dabei gilt uns als Alltagskulturforscherinnen und -forschern ein weites Verständnis von Kino, welches dieses einerseits als Erfahrungsraum betrachtet, sich andererseits nicht vor weiteren Angebotsformen der gemeinsamen Filmrezeption als sozialer Praxis verschließt. ${ }^{18}$ Der zeitliche Fokus ist auf die Gegenwart ausgerichtet und wirft über die Erzählungen der Zeitzeugen sowie historische Quellen einen Blick zurück. ${ }^{19}$ Dieser Beitrag gibt im Folgenden anhand von zwei Kinoporträts einen ersten Einblick in das Projekt und diskutiert Beobachtungen aus dem Feld.

\section{Das Corso Film Casino in Kaldenkirchen}

Ende September 2019 konnte das erste rheinische Kinoporträt über das Corso Film Casino in Kaldenkirchen veröffentlicht werden. Kaldenkirchen liegt am Niederrhein an der

16 LVR Pressemeldungen: Kinokultur im ländlichen Raum.

17 https://alltagskulturen.Ivr.de/.

18 Augenblick, 56/57 (2013); Mundhenke: Postmoderne Cinéphilie, S. 86-99.

19 Haake: Ins Kino gehen, S. 63-81. 


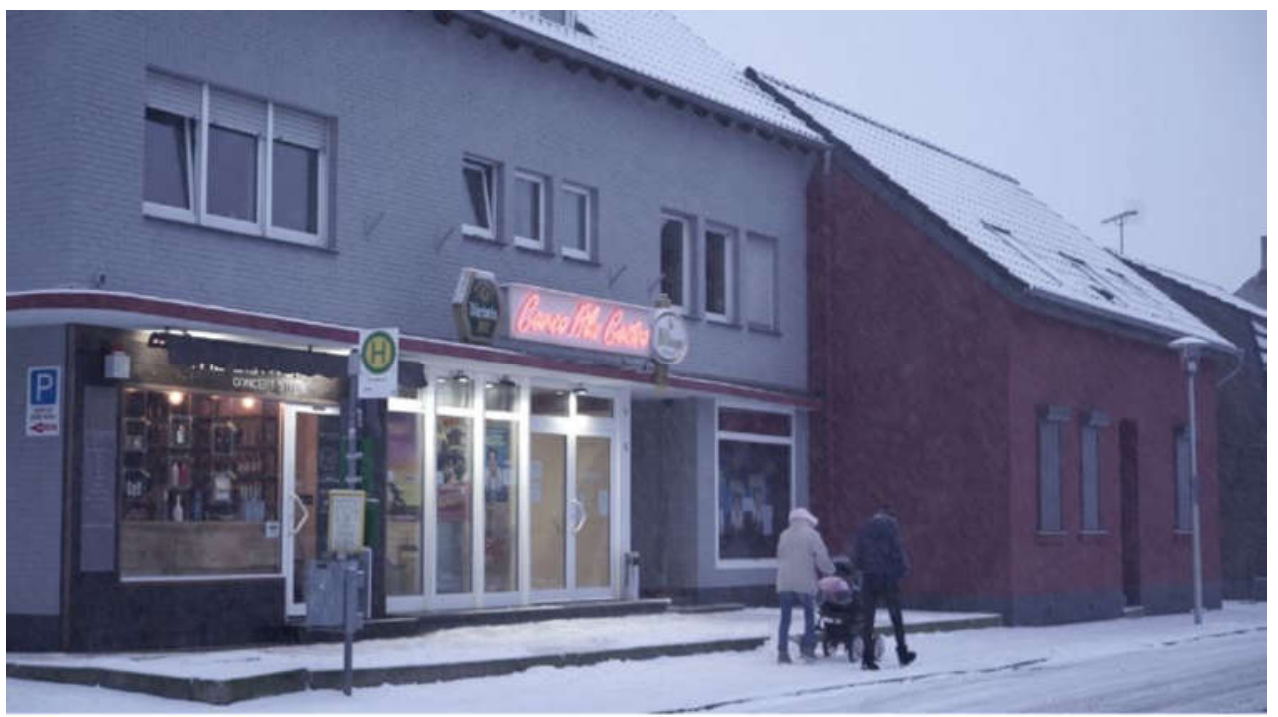

Abbildung 1: Fassade des Corso Film Casino in Kaldenkirchen (Quelle: Benda Film).

niederländischen Grenze von Nordrhein-Westfalen und hat circa 10.000 Einwohnerinnen und Einwohner. ${ }^{20}$ Seit der Gebietsreform 1970 ist die Stadt Kaldenkirchen zu einem Ortsteil von Nettetal geworden. Die Gemeinde ist umgeben vom Naturpark Maas-Schwalm-Nette, einem grenzüberschreitenden Erholungsgebiet. Die nächstgrößere Stadt ist in zehn Kilometer Entfernung Venlo in den Niederlanden. Auf deutscher Seite muss man bis ins 30 Kilometer entfernte Gladbach fahren, um ein urbanes Umfeld zu erreichen.

Im Film erzählt der Betreiber Helmut Töpfer zunächst die Geschichte des Kaldenkirchener Kinos. Sein Leben ist mit dem Kino eng verknüpft

20 https://www.nettetal.de/de/dezernat1/kaldenkirchen/\&nid1=24267. und so beginnt er seine Beziehung zum Kino anhand seiner eigenen Biografie darzustellen. Er berichtet von seinen Anfängen als Fahrradwächter während der Vorstellungen, dann von seiner Ausbildung als Filmvorführer und der damaligen Verleihlogistik der Filme per Fahrradkurier und Expresstransport mit der Bahn. Inzwischen, mit über 80 Jahren, ist es ihm ein Anliegen, die Kinotechnik an Kinder und Jugendliche im Ort zu vermitteln. Im Anschluss gibt der Film einen Einblick in das monatliche Angebot des ,Kaffee Kinos', welches besonders von Seniorinnen und Senioren besucht wird. Nach einem Meinungsbild des Publikums erläutert wiederum Herr Töpfer das Konzept seines Kinos als Servicekino. Der Kurzfilm endet mit einer Kindergruppe, die hier eine Geburtstagsfeier ausrichtet 
und begeistert von den Vorzügen ihres Lieblingskinos erzählt. ${ }^{21}$

Im Kurzfilm werden verschiedene Aspekte angesprochen, die einerseits Spezifika des Kaldenkirchener Kinos sind, aus denen sich andererseits erste Interpretationsansätze der Funktionen von Kino im ländlichen Raum entwickeln lassen. Dazu wird im Folgenden das Interview mit dem Kinobetreiber Helmut Töpfer genauer analysiert. 22

\section{Die Biografie des Betreibers}

Die Kinosozialisation von Herrn Töpfer fand im Kino Schauburg in Kaldenkirchen statt. Hier sah er seinen ersten Kinofilm: eine Tarzan-Verfilmung mit Johnny Weissmüller. Danach lernte er in den 1950er-Jahren den Beruf des Filmvorführers in diesem Einsaalkino von der Pike auf und hat seitdem alle Entwicklungen in der Kinound Vorführtechnik begleitet.

Die Vorstellungen in der Schauburg, seinem Ausbildungsbetrieb, waren meist ausverkauft. Auch als das Corso Film Casino dann 1957 als zweites Kino im Ort eröffnete, bestand keine Konkurrenzsituation, erinnert sich Töpfer. Bis zur Verbreitung der Fernsehgeräte liefen beide Kinos sehr gut. Dann kam Ende der 1960er-Jahre, wie überall, der Einbruch. Die 818 Millionen Kinobesucher im Jahr 1956 reduzierten sich bis 1967 auf 216 Millionen, so gibt es die Kommunikations- und

21 Der Kurzfilm ist abrufbar auf dem Youtube-Kanal Alltagskulturen im Rheinland, URL: https://www. youtube.com/watch?v=TV1pd-2tr_w\&feature=youtu. be.

22 Alle Informationen für die im folgenden Text paraphrasierten Aussagen von Helmut Töpfer stammen aus dem Interview des Drehteams mit ihm am 21.2.2019 in Kaldenkirchen.
Medienwissenschaftlerin Elizabeth Prommer für Deutschland an. ${ }^{23}$

Wie überall, musste man auch (oder gerade) auf dem Land erfinderisch werden, wenn man das Kinogeschäft fortführen wollte. In Kaldenkirchen konzeptionierte Herr Töpfer zu dieser Zeit (als Geschäftsführer ab 1968) die Schauburg zu einer sogenannten Filmdiskothek um - ein in den 1960er-Jahren beliebtes Konzept, bei dem das Abspielen der einzelnen Filmrollen eines Films von Tanzmusik eines DJs unterbrochen wurde. Dies zog Besucher aus ganz Nordrhein-Westfalen an.

In dieser Zeit bekamen ländliche Kinos von den Verleihern nur Filme, die bereits ein Jahr lang in den großen Städten liefen: Herr Töpfer erinnert sich an die Laufspuren der Rollen von „Spiel mir das Lied vom Tod“. Als der Besitzer 1978 in den Ruhestand ging, übernahm Helmut Töpfer schließlich das Corso Film Casino mit den 35 Millimeter Ernemann-Projektoren als Betreiber und modernisierte es seitdem stetig. Seine eigene berufliche Biografie wurde von einem enormen technischen Wandel begleitet: So erläutert Herr Töpfer im Interview, dass es heute einzig um die Bedienung des Computers gehe, die nichts mehr mit dem erlernten Beruf zu tun habe. Früher konnte er den Projektor selbst reparieren, kannte das Gerät in- und auswendig. Heute kann er an der Projektion nicht mehr als den Ton beeinflussen.

Zeitweise betrieb er parallel ein Kino und eine Diskothek im Ort. Dadurch waren ihm das Kaldenkirchener Freizeitangebot und dessen Publikum vertraut. Vor allem ist er aber in der Stadt

Prommer: Film und Kino, S. 27. 
gut bekannt. Viele Besucher nennen inn beim Vornamen und er verabschiedet regelmäßige Kinogänger mit Handschlag an der Tür. Noch heute, längst im Rentenalter, fungiert er als Theaterleiter und ist, ebenso wie seine Frau und sein Sohn, im täglichen Kinobetrieb präsent.

Viele Besucher schätzen diese familiäre Atmosphäre. Hoffentlich gibt es das noch lange, das Kino. Wir gehen nicht gern in die Städte, in die großen Kinos, das ist zu unpersönlich, zitiert Töpfer sein Publikum und erläutert weiter: Wenn zum Beispiel Kaffee Kino ist, bleiben die Leute auch noch ein bisschen sitzen, unterhalten sich über den Film. Das sind natürlich alles so Sachen, die für uns positiv sind. ${ }^{24}$

Die Filmwissenschaftlerin Franziska Heller untersuchte das Kinoerlebnis, welches als dem Fernsehen überlegener ästhetischer Rezeptionsmodus für Filme galt, vor dem Hintergrund der digitalen Heimunterhaltungselektronik und bezeichnet das Kino als kollektiven Erinnerungsort, da hier gemeinschaftliche Rezeption im öffentlichen Raum stattfindet. ${ }^{25}$ In Kaldenkirchen scheint sich die Angleichung der Erlebnisqualitäten abzuzeichnen, wenn Herr Töpfer erzählt, dass Personen ins Kino kommen, nur um Popcorn zu kaufen, da sie zu Hause einen Film ansehen möchten. Mit dieser Handlung scheint die Erlebnisqualität übertragbar zu werden.

Dem Leipziger Medienwissenschaftler Florian Mundhenke zufolge nimmt der Rezeptionsraum jedoch nach wie vor einen unterschiedlichen Stellenwert ein, wobei das Publikum heute eine größere Auswahlmöglichkeit in Bezug auf den Medienkonsum hat, mit der es situativ umgeht und zwischen Fernsehen, Internet, Mobiltelefon oder Kino wählt. Der Kinobesuch, von Mundhenke als „ikonisches Ritual“26 bezeichnet, dient wie alle weiteren individuellen Entscheidungen für ein Freizeitangebot als Distinktionsmittel und Ausdruck der Individualität in einer pluralen Gesellschaft.

Zum Zeitpunkt des Interviews stellte Herr Töpfer das Programm nicht mehr selbst zusammen, die Filmdisposition für das Corso Film Casino wurde an eine Agentur übertragen. Doch ist es inm als Theaterleiter wichtig, die Filme zu kennen. Jeden Film, der im Corso Film Casino läuft, hat er sich angeschaut. Und mit seiner Meinung darüber, ob sich ein Film mit entsprechender Altersfreigabe tatsächlich für Kinder dieses Alters eignet, hält er sich an der Kinokasse gegenüber den Eltern nicht zurück. Helmut Töpfer bezieht sich mit der folgenden Aussage auf den Film "Club der roten Bänder": Da sind Szenen drin, die sind nichts für Sechsjährige und wenn dann ein Elternpaar kommt, dann sagen wir denen das ich mein' wir sind zwar kommerziell, wir wollen Geld verdienen - aber ich seh' das etwas anders. Vielleicht ist das auch ein Grund, warum bei uns das Kino läuft. Dann sag ich zu denen: ,Das hat keinen Zweck für das Kind, lasst das lieber den Film nicht gucken.' Das glauben die Leute dann auch. ${ }^{27}$

In Situationen wie dieser tritt Herr Töpfer als Experte auf. Denn seine Persönlichkeit und seine Ideen prägen das Kaldenkirchener Kino seit den 1960er-Jahren und sind für die Besucherinnen und Besucher mit diesem Ort verknüpft. Das Corso Film Casino wird als Familienbetrieb
24 Interview Helmut Töpfer, Timecode: 00:47:18.

25 Heller: Erfahrungsraum Kino, S. 190-191.
26 Mundhenke: Von den Grenzen, S. 215.

27 Interview Helmut Töpfer, Timecode: 00:44:18. 
wahrgenommen. Die Kinoerinnerung der Generation der heute erwachsenen Kaldenkirchener Bevölkerung ist durch dieses Kino geprägt. Die Medienwissenschaftlerin Susanne Haake thematisiert in ihrer Dissertation die Dynamik dieser Erinnerung, die generationenabhängig ist und mit der jeweiligen Konstruktion von Jugend korreliert. Ihre Forschung verweist auf kollektive Erfahrungen der Befragten, die mit gesellschaftlichen Aushandlungsprozessen, in ihrem Beispiel dem zeitgenössischen Jugendschutz, in Zusammenhang stehen. ${ }^{28}$

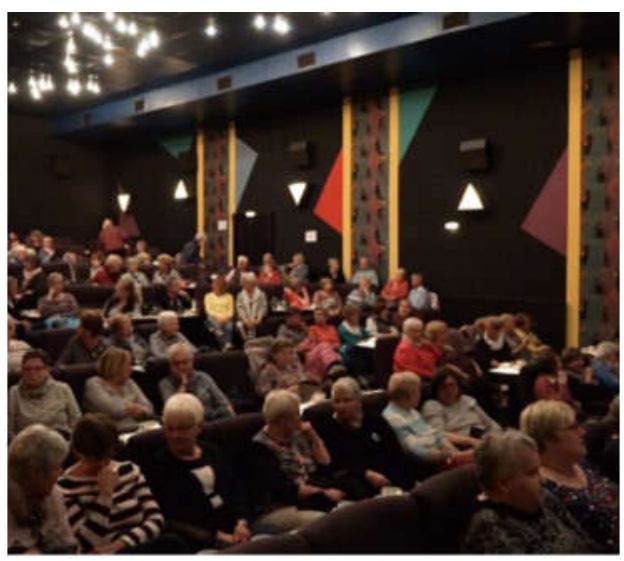

Abbildung 2: Kinopublikum beim Kaffee Kino im Corso Film Casino (Quelle: Benda Film).

\section{Das Kino als Begegnungsraum}

Mit seinem Konzept schafft Herr Töpfer einen Raum für Begegnung in Kaldenkirchen. Die unterschiedlichen Angebote wie das ,Kaffee Kino', ,Ladies First', die Übertragungen von

28 Haake: Ins Kino gehen, S. 80.
Spielen der Fußballweltmeisterschaft oder die Ausrichtung von Kindergeburtstagen fördern die Kommunikation und das gemeinsame Filmerleben. Dieses Konzept des Kinos zwischen Wohnzimmer und Eventlocation, das Florian Mundhenke zufolge eine Implosion des Kinos darstellt, ${ }^{29}$ bringt Helmut Töpfer auf den Punkt, wenn er auf die Breite der Anfragen zur Nutzung seines Kinosaals verweist:

Von der Hochzeit bis zur Goldhochzeit, die wollten 'nen ganz alten Film gucken mit Roy Black oder so, wir nehmen auch alles an. Kommt einer und hat Geburtstag und fragt: ,Kann ich das Kino mieten? Ich möchte mit meinen Freunden einen Film gucken.' Geschlossene Gesellschaft, dann gucken die hier eine DVD. Wir können alles abspielen.

Das Kino als Erlebnisraum verspricht per se eine außeralltägliche Erfahrung. Neben dem Film und dessen Narrationsmustern spielt hier das Raumerleben eine zentrale Rolle. Durch die besondere Atmosphäre des dunklen Saals, die Anwesenheit der weiteren Zuschauer und des laufenden Films wird laut Filmtheoretiker Christian Metz „beim Zuschauer [ein] Prozeß der ,Partizipation' aus[gelöst], der in gleicher Weise die Wahrnehmung und die Gefühle betrifft". ${ }^{30}$ Andreas Hepp und Waldemar Vogelsang ergänzen, dass das Kino ein „bewährter Erlebnisraum, [...] [sei], um die gewünschten Stimmungen und Gefühle auszulösen." ${ }^{31}$

Das Konzept des Servicekinos ermöglicht darüber hinaus ein besonderes Erlebnis, indem die Besucherinnen und Besucher per Tischtelefon

29 Mundhenke: Von den Grenzen, S. 213

30 Hepp/Vogelsang: Kino als Medien-Event, S. 244.

31 Hepp/Vogelsang: Kino als Medien-Event, S. 242. 
Eis- und Getränkebestellungen durchgeben können, die ihnen dann am Platz serviert werden ein Konzept, das Wohnzimmeratmosphäre ins Kino bringt, wodurch das Corso mehrmals unter die 100 besten deutschen Kinos gewählt wurde. Dieser Erfolg des Corso Film Casinos lässt sich mit den Soziologen Winfried Gebhardt, Ronald Hitzler und Michaela Pfadenhauer als Reaktion auf allgemeine Entwicklungen einer Eventisierung des Freizeitangebots in der Erlebnisgesellschaft deuten. ${ }^{32}$ Denn was das Corso Film Casino an Service bietet, geht deutlich über ein ,normales' Kinoerlebnis hinaus. ${ }^{33}$ Dabei bedienen die Wählscheibentelefone, die besonders gern vom jungen Publikum genutzt werden, einen besonderen Retro-Charme, der in den letzten Jahren populär geworden ist.

Im filmischen Porträt wird auf die Kindergeneration als zukünftiges Publikum fokussiert und auf eine bestimmte Erfahrung, die Menschen im Kino machen und die sie zu regelmäßigen Kinogängerinnen und -gängern werden lässt. Interesse an der Technik attestiert Herr Töpfer den Kindern und erklärt ihnen deren Entwicklung, die er selbst von den ersten Ernemann-Projektoren bis zur heutigen 4k-Projektion miterlebte. Auf diese Weise gibt er seine Faszination für das Kino weiter und damit auch einen Teil seiner Lebensgeschichte. Technikfaszination als ein Narrativ der Kinoerinnerung weist auch Susanne Haake - neben dem zum Kino zurückgelegten Weg, dem Filminhalt sowie der Verzauberung - für die von ihr beforschte Generation aus, die in den

\footnotetext{
32 Mit dem Begriff Erlebnisgesellschaft beziehe ich mich auf Schulze: Erlebnisgesellschaft.

33 Gebhardt/Hitzler/Pfadenhauer (Hg.): Events, S. 18.
}

1930er- bis 1950er-Jahren ihre Kinosozialisation im ländlichen Saarland erfuhr. ${ }^{34}$

Diese Förderung der Kinder sieht Töpfer auch als Investition in die Zukunft des Kinos im ländlichen Raum, um eine neue Generation an das Kino zu binden. 25 Jahre lang beteiligte er sich an Aktionen zum Weltkindertag, dazu verkaufte er vergünstigte Eintrittskarten an Geschäftsleute, die diese an Kinder weiterverschenkten. Noch heute kommen Erwachsene auf ihn zu und erinnern sich an ihr erstes Kinoerlebnis, sagt Helmut Töpfer: Wie oft ich das heute noch hör, von Erwachsenen: Wir sind als Kinder schon hier im Kino zum Weltkindertag gewesen' ${ }^{35}$

Mit Zukunftsprognosen hält sich Herr Töpfer jedoch zurück: Jederzeit könne es vorbei sein, aber das Kino sei schon oft totgesagt worden. 40 Prozent sei die durchschnittliche Auslastung der Vorstellungen und mit der Gastronomie könne man den Umsatz bestreiten, mit den Eintrittskarten, von deren Erlös 50 Prozent an den Verleiher gehen, nicht. Die Filme werden nach kommerziellen Gesichtspunkten ausgewählt. Trotzdem kann mal ein Flop dabei sein oder ein heißer Sommer, wie 2018, als die Besucherzahlen stark zurückgingen.

Über die Jahrzehnte halten konnte sich das Kino zum einen, weil es das einzige im Ort ist, zum anderen wegen des Serviceangebots, neuester Technik, der besonderen Sessel und der damit verbundenen großen Beinfreiheit. In den anderen Ortsteilen Nettetals, Hinsbeck, Breil und Lobberich, schlossen die Kinos nach und nach, erzählt Helmut Töpfer im Interview. Von auswärtigen Kinobesuchern hört er immer wieder, wie

\footnotetext{
34 Haake: Ins Kino gehen, S. 65.

35 Interview Helmut Töpfer, Timecode: 00:36:50.
} 
besonders sein Kino sei. Wünschen würde sich Herr Töpfer eine Unterstützung durch die Stadt, die doch ein Interesse daran haben sollte, diesen Ort zu erhalten. Inwieweit das Corso Film Casino die Schließung aufgrund der Corona-Pandemie verkraftet, kann in der aktuellen Situation nicht eingeschätzt werden. Derzeit informiert die Internetseite mit folgendem, zuversichtlich klingenden Text: Liebe Kinofreunde, aufgrund der aktuellen Vorkommnisse unterbrechen wir ab sofort unseren Spielbetrieb laut Beschluss der Landesregierung NRW bis auf weiteres! Wir wünschen allen Gästen des Corso Film Casino alles Gute und hoffen, dass schnellstmöglich alles wieder seinen normalen Weg geht! Mit besten Grüßen, euer CFC Team.

\section{Auf Basis der Analyse des Interviews mit Herrn Töpfer kann auf folgende Eigenschaften und Funktionen des Kinos in Kaldenkirchen ge- schlossen werden:}

- Das Kaldenkirchener Kino zeichnet sich durch die Persönlichkeit seines Betreibers aus: a) Helmut Töpfer ist ein alter Hase, eine Instanz im Ort, seine Biografie ist eng verknüpft mit der lokalen Kinogeschichte, er ist präsent, eher ein Lokal- als ein Kulturunternehmer. Der Kinobetrieb wird ergänzt durch Service im Saal und Events im familiären Rahmen. b) Herr Töpfer nimmt die Position eines Experten ein, der dank seiner Medienkompetenz Empfehlungen und Beratung hinsichtlich der Eignung von Filmen für spezifische Altersgruppen anbietet. Er ist eine Vertrauensperson, deren Meinung von den Besucherinnen und Besuchern geschätzt wird.

- Für die Ortschaft übernimmt das Kino eine wichtige Funktion als Begegnungsraum für die verschiedenen Generationen. Das Kino bietet nicht nur anonymen Filmkonsum, sondern Raum für Kommunikation und Diskurs.

- Die Übernahme erweiterter lokaler Funktionen, die im ruralen Raum nicht abgedeckt sind, für die aber ein großer Bedarf da ist, ist eine Überlebensstrategie des Kinos.

- Bei der Programmauswahl wird wenig experimentiert, da es den Geschmack aller Altersgruppen abdecken muss.

- Der Kinosozialisation kommt eine Schlüsselaufgabe zu. Die zukünftige Generation wird bewusst adressiert, um das Erlebnis Kino früh zu verankern und diese als Publikum zu binden.

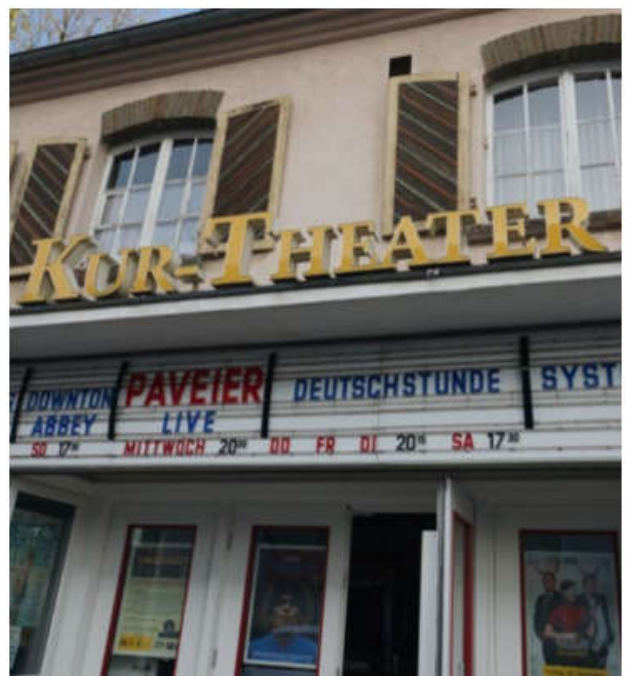

Abbildung 3: Fassade des Kur-Theaters in Hennef (Foto: Robin Stecken). 


\section{Das Kur-Theater Hennef}

Das zweite Kinoporträt beschäftigt sich mit dem Kur-Theater in Hennef, welches sich durch sein Bühnenprogramm, ergänzt durch Kabarett und Konzerte, eher als Kulturzentrum versteht.

Die Stadt Hennef liegt mit rund 48.000 Einwohnern im Rhein-Sieg-Kreis, circa 14 Kilometer von Bonn entfernt, und besteht aus 89 Ortschaften. Hennef ist deutlich weniger ländlich gelegen als Kaldenkirchen und mit dem öffentlichen Personennahverkehr gut angebunden. Es liegt an der Peripherie des rheinischen Ballungsgebietes Köln/Bonn. Das Kino Kur-Theater befindet sich seit 1938 im Zentrum Hennefs in einem denkmalgeschützten Gebäude. Es ist das letzte von vormals drei Hennefer Kinos.

Im Kurzfilm erzählen die beiden Programmverantwortlichen Petra Stratmann und Daniel Huys, wie sie die Auswahl der zu zeigenden Filme treffen, dabei setzt das Kur-Theater auf eine alle Altersgruppen und verschiedene Geschmacksrichtungen ansprechende Programmmischung und die Einbindung lokaler Vereine sowie Sonderveranstaltungen. ${ }^{36}$ Die Arbeit von Frau Stratmann und Herrn Huys ist ehrenamtlich, da das Kino seit der Aufgabe durch die Betreiberfamilie 2003 als Verein weiterbesteht. Huys ist dem Kino bereits seit seiner Jugend verbunden und gehört zu den Gründungsmitgliedern des eingetragenen Vereins Kur-Theater Hennef, der inzwischen circa 1.400 Mitglieder hat. Neben den ehrenamtlich Tätigen beschäftigt das Kur-Theater junge Menschen als Minijobberinnen und

36 Der Kurzfilm ist abrufbar auf dem Youtube-Kanal Alltagskulturen im Rheinland, URL: https://www. youtube.com/watch?v=er81gdb/Upg. -jobber, so erzählt das Vorstandsmitglied Ingo Teusch. Die Hennefer Bürgerinnen und Bürger sowie Betriebe engagieren sich für den Erhalt und die Renovierung ihres Kinos. Der Filmvorführer Sebastian Binz empfindet sein Hobby als Entspannung vom Beruf, indem er anderen Menschen einen schönen Abend bereitet. Im Folgenden werden die weiterführenden Interviews mit den Betreibenden auf die Funktionen des Kinos im Ort hin analysiert.

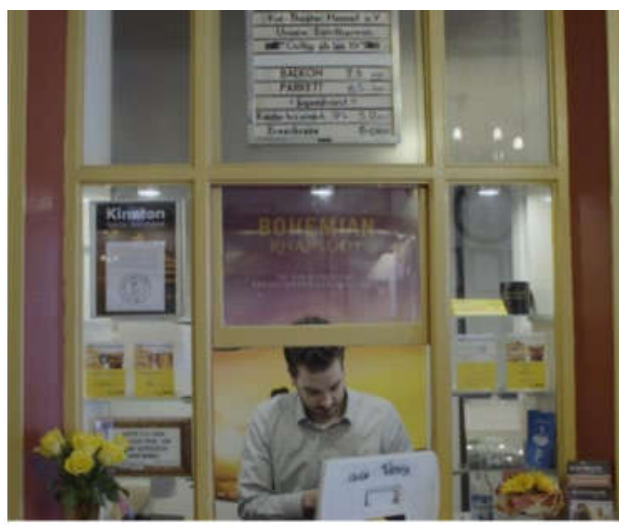

Abbildung 4: Kassenbereich des Kur-Theaters in Hennef (Quelle: Benda Film).

\section{Die Gemeinschaft der Kinomacherinnen und -macher}

Herr Huys ist mit dem Kur-Theater verbunden, seit er als Fünfzehnjähriger erstmals dort als Gast den Film „Dangerous Minds“ sah. Kurz darauf trat er in dem damaligen Familienbetrieb einen Schülerjob an und bekam Einblicke hinter die Kulissen der Kinoarbeit, die inn seitdem nicht mehr losließ. Mit dem Ort verbindet inn persönlich viel. Als die vormaligen Betreiber aus wirtschaftlichen Gründen das Kino aufgeben 
mussten, unterstützte Herr Huys gemeinsam mit Anderen die Idee, das Kino als Verein zu übernehmen. Obwohl er seit etlichen Jahren nicht mehr in Hennef wohnt, hat er nie überlegt, mit der Vorstandsarbeit im Verein aufzuhören. Herr Huys bedauert es, nicht mehr so häufig vor Ort sein zu können, verfolgt jedoch die Besucherzahlen über eine abendliche Benachrichtigung auf sein Mobiltelefon. Das Engagement für das Kino ist seine Feierabendund Wochenendbeschäftigung: Es ist über die vielen Jahre so in den Alltag integriert, dass ich halt weiß: Ok, Montagmorgens musst du überlegen was läuft ab Donnerstag, Dienstag wird der Newsletter rausgeschickt, und so weiter und so weiter. ${ }^{37}$ Ihn motiviert besonders, dass der Verein das Kino inzwischen bereits 16 Jahre lang erfolgreich betreibt. Finanzielle Unterstützung von Stadt oder Land erhält das Kino nicht. Es fınanziert sich über die Mitgliedsbeiträge, über Karten-, Getränke- und Snackverkauf sowie, mit ein bisschen Glück, den Kinoprogrammpreis der Filmstiftung, für den es sich jährlich bewirbt. Das Hennefer Kino muss als Verein keinen Gewinn machen und steht somit nicht unter dem wirtschaftlichen Druck anderer Kinos, deren Betreibende von den Einnahmen leben müssen. Petra Stratmann sagt zur Begründung ihres Engagements: Man muss Kino schon lieben. ${ }^{38}$ Und nennt die Mitarbeit an der Programmgestaltung eines Kinos als einen von ihr lange gehegten Wunsch. Sie ist nach Hennef zugezogen, gehörte zum Stammpublikum und wuchs dann in den Verein hinein.

37 Interview Daniel Huys, Timecode: 00:37:13.
Filmgeschmack, Geschlecht und Alter der beiden Programmverantwortlichen Frau Stratmann und Herr Huys unterscheiden sich, das sei gut für die Filmauswahl, denn sie werde dadurch breiter. Dazu Frau Stratmann: Und dadurch, dass ich viel älter bin und auch einen ganz anderen Geschmack hab, ist das bei uns sehr abwechslungsreich. So wie, Ich war noch niemals in New York', da weiß ich genau, den findet er [Herr Huys] total scheiße und ich find' den gut. Das ist einfach super, dass wir uns da so ergänzen und jeder auch mal 'nen anderen Film... zum Beispiel ,Joker', mein Lebtag würde ich da nicht reingehen. Das macht es einfach spannend bei uns. ${ }^{39}$ Die Vorstandsmitglieder haben sich die Aufgaben untereinander aufgeteilt, somit gibt es weitere Personen, die für die Finanzen, die Technik oder das Kulturprogramm zuständig sind. Es handelt sich um ein eingespieltes Team von etwa 15 Personen, die auf freiwilliger Basis im Kino helfen. Den Hauptvorstand hat der Verein um sechs Beisitzende erweitert, um hier mehr Aufgabenbereiche abdecken zu können. Die Tätigkeiten greifen ineinander, es arbeiten allerdings alle selbständig und übernehmen Verantwortung auch für unattraktive Aufgaben: Da muss man auch jemanden für haben, der sagt: ,Ja, ich mach das', und das sind jetzt echt nicht die glamourösen Jobs im Kinobereich zu sagen: ,Ich mach hier 'ne Abrechnung', oder die Löhne überweisen. Das ist alles nicht so sexy. ${ }^{40}$ Trotzdem gebe es keine Konkurrenz, ergänzt Herr Teusch, denn die gemeinsame Motivation sei der Erhalt des Kinos, hier sieht er positiv in die Zukunft.

39 Interview Petra Stratmann, Timecode: 00:24:12.

40 Interview Daniel Huys, Timecode: 00:58:55. 
Eine zukünftige Aufgabe sieht Frau Stratmann in der Begeisterung des Nachwuchses für das Kino als Publikum, jedoch auch in Bezug auf die Vereinsarbeit. Über die angebotenen Minijobs an der Kasse oder im Service, die besonders von Jugendlichen besetzt werden, erreicht der Verein die junge Zielgruppe auch als dauerhafte Vereinsmitglieder. Auf diese Weise entsteht eine Bindung zum Kino.

Mit Blick auf die Geschichte von Kino allgemein, das bereits mehrmals totgesagt wurde, hat Herr Huys keine Zweifel, dass dieses weiterbestehen wird, trotz technischer Neuerungen, prophezeitem Publikumsschwund oder Streamingdiensten:

Dieses Erlebnis Kino, das hat sowas Besonderes, dass das nicht an sich gefährdet ist und ich sehe auch nicht mehr so die Schwierigkeit durch Konkurrenz. Wie jetzt Multiplex in der Nähe oder so, weil ich einfach die Erfahrung mache, jetzt seit sechzehn Jahren, das kann alles nebeneinander existieren, das kann alles funktionieren. Wo ich tatsächlich 'ne Gefahr sehe: Jetzt fügt sich das ja als Verein so lange schon zusammen im Vorstand und das hängt viel an dem Engagement von einzelnen Personen. Es ist schon auch möglich mal jemanden zu ersetzen aber würde sich im Vorstand ganz groß was ändern, würden die entscheidenden Positionen wegbrechen und man kriegte das nicht so schnell nachbesetzt, das wäre schon echt ein Problem. ${ }^{41}$

\section{Ein Kino für die Bevölkerung}

Die Geschichte des Kur-Theaters ist die eines typischen Kinoortes. Das Gebäude wurde 1938 für den Kinobetrieb erbaut. Vorausgegangen war das mobile Kino der Besitzerfamilien in der eigenen Gaststätte. Der Bau wurde mit Bühne, Orchestergraben und 500 Sitzplätzen konzipiert. In den vergangenen 80 Jahren wurde es teilweise als Opernhaus genutzt, dann rückgebaut und nur noch als Kino betrieben, bis inm 2003 das Aus drohte. Die Gründungsmitglieder des Vereins Kur-Theater Hennef reagierten auf einen Zeitungsartikel, der die Schließung thematisierte. Der Verein baute eine Bühne ein, seit Oktober 2003 wird hier zusätzlich wieder Kulturprogramm angeboten. Von 2003 bis 2019 fanden circa 400 Veranstaltungen statt. Das seien deutlich mehr als die Stadt selber macht und ich denke die Stadt kann froh sein, dass es das KurTheater in Hennef hier gibt, so Herr Teusch. ${ }^{42}$ Seit 2013 verfügt das Kino zudem über eine digitale Projektionsanlage. Das Haus wird als Denkmal erhalten, die technische Ausstattung ist allerdings modern.

Die Mitgliederzahl des 2003 für den Erhalt des Kinos gegründeten Vereins lag zunächst bei circa 40 Personen, derzeit liegt sie bei 1.400 Personen. Dies verweist auf ein großes Interesse an dessen Unterstützung. Einen Grund dafür sehen die Kinobetreiberinnen und -betreiber in den Erinnerungen, die viele Hennefer Bürgerinnen und Bürger mit diesem Kino verbinden. Herr Huys meint: Die Hennefer wüssten es wieder zu schätzen, dass es noch ein Kino gibt. Und dass es ein besonderes Kino ist. Und das merk' ich

42 Interview Ingo Teusch, Timecode: 00:08.32. 
an Mails und so weiter. Oder es gibt ja auch diese ganzen Googlebewertungen. Ich komm aus dem Kino und dann wird man direkt gefragt: Wie fandest du es? Möchtest du eine Bewertung abgeben? Und wir haben da sehr sehr gute Bewertungen, du, da gibt es total nette Kommentare. ${ }^{43}$ Die Betreiberinnen und Betreiber erfahren Wertschätzung von der Hennefer Bevölkerung. Zur Zeit der Vereinsgründung musste viel repariert und umgebaut werden und neben der Eigenleistung der Mitglieder wurde der Verein dabei von lokalen Betrieben unterstützt. Den Einbau der Bühne für das Kulturprogramm und auch die Dachreparatur stemmten lokale Handwerksbetriebe, denen das Kino am Herzen liegt. Diese Unterstützung interpretiert Herr Huys als typisch für einen kleinen Ort, in dem eine soziale Verbindlichkeit herrscht. Begründet wird diese mit der Nostalgie, mit den eigenen Erinnerungen der Helfenden an ihre Erlebnisse in diesem Kino. Das Kur-Theater Hennef gilt als Inbegriff des Kinos, so diente es auch als Kulisse für den Videodreh zum Lied „Wenn et Leech usjing em Roxy“ der Kölner Band Bläck Fööss.

In Zusammenhang steht diese positive Konnotation des Hennefer Kinos auch mit dem Gebäude, welches einen besonderen Charme ausstrahlt: Das ist ein besonderer Saal und dadurch hat das auch so'n Eventcharakter ins Kino zu gehen. Ich mein', wenn das jetzt ein schäbbigeres Kino in so einer 70er-Jahre Optik wäre, hätte man vielleicht auch nicht so viel emotionale Bindung dazu gehabt und gesagt wir wollen es erhalten. Dass es so diesen Retrocharme mit ausstrahlt, es ist ja von '38, alles ein bisschen durcheinander, du

43 Interview Daniel Huys, Timecode: 00:43:14. kennst dich besser aus [zu Frau Stratmann], es ist glaub ich in so Richtung so 50er, ist ja auch nicht alles originalgetreu. Petra Stratmann ergänzt: Aber allein die Sitze haben wir selbst aufgepolstert, die sind nicht so neu, das macht es einfach insgesamt. Dazu erneut Herr Huys: Da hatten wir auch so Angebote für diese Plastikbombersitze und dann sagte einer aus dem Verein: ,Das können wir machen, dann müssen wir aber noch Raufaser tapezieren'. Stattdessen, und das war natürlich wieder sauviel Arbeit, wurde hier mit so einem verrenteten Restaurateur und ganz viel Eigenleistung, wurden alle Sitze neu bezogen. Die müssen dann wieder feuerfest sein und was man dann alles zusätzlich berücksichtigen und bedenken muss. ${ }^{44}$

Die Mitgliedsbeiträge hält der Verein bewusst gering, um für Interessierte keine Hürden zu schaffen. So kann eine ideelle Bindung vieler Förderer an das Kino gewährleistet werden. Für den Verein wird zudem viel geworben. Ebenfalls ist es dem Verein ein Anliegen, auch die Eintrittspreise für die Vorstellungen niedrig zu halten, so Herr Teusch: Uns ist es lieber wenn eine Familie noch mit zwei oder drei Kindern ins Kino gehen kann und sich das leisten kann mit Popcorn. ${ }^{45}$ Auf diese Weise gibt das Kino die Wertschätzung zurück.

Herr Huys ist seit 2003 für das Programm verantwortlich, mit Frau Stratmann ist seit 2017 eine weitere Person dazugekommen, die sich vor allem um das Sonderprogramm kümmert. Frau Stratmann hält Kontakt mit Seniorenzentren, pflegt eine Kooperation mit der Volkshochschule, weitere Partner sind die

44 Interview Daniel Huys, Timecode: 00:49:29.

45 Interview Ingo Teusch, Timecode: 00:07:25. 
Stadtbücherei, der Naturschutzbund oder der örtliche Fußballverein, der die Dokumentation über Diego Maradona in einer Sondervorführung sehen möchte. Ohne Anbindung an einen Verein oder eine Veranstaltung, die wiederum Publikum ins Kino bringt, könnten bestimmte Filme, zum Beispiel Dokumentationen, nicht gezeigt werden. Diese Kommunikationsarbeit kostet Zeit und persönlichen Einsatz. So organisiert Frau Stratmann einen Fahrdienst, damit betagtes Publikum zum Kino kommt, oder informiert auf dem örtlichen Weihnachtsmarkt über die Arbeit des Kinovereins. Neben dem Angebot für Seniorinnen und Senioren - hier erklärt Frau Stratmann: Seniorenkino ist total gut angekommen. Einlass ist um zwei, da stehen die da schon hufescharrend vor der Tür und überschwemmen uns dann - bietet das Kur-Theater ein Wickeltaschenkino für Eltern mit kleinen Kindern an: Wir haben zwei Seniorvorführer und ohne die könnten wir das gar nicht machen. Wir sind ja ein Verein, alle anderen Mitglieder sind halt berufstätig, Schüler oder Studenten und die haben sich da richtig gut eingearbeitet und dann bieten wir das halt an. Da gibt's Brötchen auch und das ist für junge Eltern, aber es kommen auch viele andere, die einfach sagen: , Mir ist es zu spät abends.' Und so gucken wir einfach, wo kriegen wir hier so Nischen, wen erreichen wir. Und mein Ziel wäre, noch viel mehr junge Leute ins Kino zu holen und das ist halt schwierig heute mit diesen Streamingdiensten und da basteln wir auch noch an 'nem Programm. ${ }^{46}$

Um sich nicht einzig auf seinen eigenen Filmgeschmack verlassen zu müssen, zieht Daniel
Huys die Filmkritik in der Cinema hinzu oder schaut Trailer neuer Filme an. Da das Kur-Theater kein Erstaufführungskino ist, kann der Spielerfolg von Filmen auch bei der Konkurrenz beobachtet werden, um dann zu entscheiden, ob der Film auch im eigenen Haus gespielt werden soll. Nur in Ausnahmesituationen wie beispielsweise bei den "Star-Wars-" oder "James-Bond"Filmen nimmt das Kur-Theater einen Film ab dem Filmstart ins Programm. Daran ist zumeist ein Vertrag gekoppelt, diesen Film drei Wochen lang spielen zu müssen; für ein Einsaalkino stellt das eine enorme Einschränkung der Programmgestaltung dar.

Oftmals wird Herr Huys auch durch sein Publikum auf interessante Filme aufmerksam gemacht. An seine Aufgabe hat er den Anspruch, eine gründliche Vorauswahl zu treffen: Wichtig finde ich eben, wenn die Leute hierhin kommen, die sollen davon ausgehen können: Ich bekomme hier keinen Schrott gezeigt. Da macht nicht irgendwer das Programm, Hauptsache so und so viel Vorstellungen sind abgedeckt. Auch wenn ich mir die Filme vorher nicht alle angucken kann. Aber ich will mich schon so informieren und davon ausgehen, dass wir da was Gescheites zeigen. ${ }^{47}$ Diese Vorauswahl sieht er auch als Vorteil des Kinos gegenüber Streamingdiensten, in deren großem Angebot sich viele Menschen nicht orientieren könnten. Die Strukturierung des Programms und eine Qualitätskontrolle nimmt das Kino dem Publikum ab.

Das Kur-Theater steigerte seine Publikumszahlen entgegen jeder Prognose und der Entwicklung der Kinobranche im Jahr 2019 deutlich. 
Herr Huys erklärt, dass dies bereits mit der Entscheidung für das Spielen sehr erfolgreicher Filme, wie 2019 „Der Junge muss an die frische Luft“ oder "Bohemian Rhapsody“, in Zusammenhang stehen kann. Das Publikum des Kur-Theaters kommt dabei aus einem weiteren Umkreis Hennefs. Mit dem nächstgelegenen MultiplexKino in Siegburg steht es aufgrund seines differenzierteren Programms nicht in Konkurrenz. Um das Überleben des Kinos machen sich Frau Stratmann und Herr Huys keine Sorgen, solange sich das Kino auf seine Qualitäten besinne (besonderer Ort, gemeinsames Erlebnis, Mehrwert zusätzlich zum Film) und nicht nur die Abdudelstation ${ }^{48}$ für Comicverfilmungen und Disney-Realverfilmungen sei, denn genau hier ist laut den Hennefer Programmverantwortlichen ein neuer Impuls gefragt.

\section{Zur Strategie des Kinomachens in Hennef können folgende Punkte zusammenfassend festgehalten werden.}

- Das Kur-Theater in Hennef zeichnet sich einerseits durch das Gebäude aus:

- Durch das denkmalgeschützte Bauwerk, dem ein historischer Charme zugeschrieben wird, erhält der Wunsch nach dem Erhalt des Kinos über die Architektur eine weitere Dimension.

- Die lange Betriebszeit des Ortes als Kino führt dazu, dass hier über Jahrzehnte die kollektive Kinosozialisation der Hennefer Bevölkerung stattfand und zahlreiche Personen den Ort mit individuellen Erinnerungen verbinden.

48 Interview Daniel Huys, Timecode: 01:00:30.
- Beides begründet die hohe Zahl an Mitgliedern im Förderverein. Die Unterstützung eines guten Zwecks bedeutet soziales Prestige und ist dem eigenen Ansehen zuträglich. So werden diverse Protagonistinnen und Protagonisten, die teils selbst relevante Positionen im Ort besetzen, an das Kino gebunden. Dieses Netzwerk lässt sich aktivieren, geht es um Unterstützung bei beispielsweise handwerklich-baulichen Tätigkeiten.

- Weiter zeichnet sich das Kur-Theater durch die Vereinsstruktur sowie die Veranstaltungsplanung aus: Durch die stetige Einbindung von weiteren lokalen Akteurinnen und Akteuren wie Vereinen, Seniorenzentren und Bildungseinrichtungen in die Programmplanung verfügt das Kur-Theater über Rückhalt in der Bevölkerung sowie ein gutes Netzwerk, es wird dadurch zu einem Kino von Bürgerinnen und Bürgern für und mit Bürgerinnen und Bürgern.

- Hinter der Vorstandsarbeit steht das Engagement einer kleinen Gruppe ehrenamtlich Tätiger, die sich als erfolgreich handelnde Gemeinschaft verstehen und stolz auf ihre Leistungen sind.

- Durch das Angebot von Kino und Kulturveranstaltungen im Bühnenprogramm schließt das Kur-Theater eine städtische Versorgungslücke und hegt daher keine Existenzängste.

\section{Fazit und Ausblick}

Die untersuchten Kinos in Kaldenkirchen und Hennef unterscheiden sich durch ihre räumliche Lage und somit die infrastrukturellen Merkmale der Gemeinden und deren soziale Struktur. Bei 
Kaldenkirchen handelt es sich um einen Familienbetrieb, in Hennef um ein vereinsgeführtes Kino. Beide sind in ihrem Ort als letzte Kinos übriggeblieben.

Durch die Analyse der Interviews konnten Strategien der Betreibenden herausgearbeitet werden, die auf Funktionen des Kinos im ländlichen Raum verweisen: Obwohl in den beiden Kinoporträts der Blick auf sehr unterschiedlich geführte Lichtspielhäuser gerichtet wurde, gibt es Gemeinsamkeiten. Das Kino spiegelt seinen Standort im Rheinland wider. Denn die Programmauswahl ist abhängig von Region, Lage, dem Publikum und davon, ob das Kino als Wirtschaftsunternehmen geführt wird. Hier verfügt das Kur-Theater in Hennef über eine größere Freiheit als das Corso Film Casino. Im ländlichen Raum besteht das Kinopublikum dem demographischen Wandel zufolge vermehrt aus sehr jungen und älteren Besucherinnen und Besuchern, ${ }^{49}$ wie an den Angeboten für diese Zielgruppen in Kaldenkirchen und Hennef ablesbar ist. Die Kinobetreibenden an beiden Standorten kennen ihr Publikum gut und stimmen ihr Angebot passgenau ab.

Das Kino abseits der Großstadt ist stets ein sozialer Ort des Austausches zwischen Betreibenden und Publikum und nie ein Ort des reinen Filmkonsums. Die Betreiberinnen und Betreiber sind persönlich ansprechbar und pflegen Netzwerke. Sie binden über Sonderprogramme verschiedene Bevölkerungsgruppen ein. Im Kino spielt sich viel mehr ab als Filmprogramm, denn das Kino zählt als lokaler Veranstaltungsraum für Menschen jeglichen Alters.

49 Bald: Kino, S. 40
Die Kinomacherinnen und -macher gehen ihrer Aufgabe mit Leidenschaft und großem Engagement nach. Das ,Kinomachen' ist nicht nur ein Beruf oder Hobby, sondern eng mit der eigenen Biografie verknüpft. Die Funktion des Kinos als Ort des sozialen Erlebens markiert einen wichtigen Unterschied zur Konkurrenz der OnlineStreamingdienste. Nicht der reine Filmkonsum steht im Kino im Vordergrund, sondern das gemeinschaftliche Erlebnis. Über Sonderprogramme, Diskussionen mit Filmemacherinnen und -machern oder Vortragsveranstaltungen wird ein Mehrwert geschaffen und das Kino als kultureller Ort sichtbar. Die beiden untersuchten Beispiele zeigen, dass die Bindung des Publikums an das jeweilige Kino durch die Betreiberinnen und Betreiber aktiv hergestellt wird..$^{50}$ Die dadurch produzierte Identifikation des Publikums mit ,seinem' Kino erweist sich damit als Überlebensstrategie. Es wird sich zeigen, inwiefern dies kleinen Kinos im ländlichen Raum auch über die Corona-Krise hinweghelfen kann, die viele vor existenzielle Probleme stellen wird. Erste Spendenaufrufe und Crowdfunding-Kampagnen zur Rettung ,seines' Kinos deuten darauf hin, dass die Identifikation des Publikums mit dem jeweiligen Kino zumindest einen Teil zum wirtschaftlichen Überleben des Kinos beitragen kann. So hat beispielsweise das Werbeunternehmen Weischer.Cinema die Kampagne hilfdeinemkino gestartet. Über eine Deutschlandkarte kann das eigene Stammkino ausgewählt werden. Neben der Möglichkeit direkt zu spenden,

50 Dies nennt ebenfalls Katja Bald als Teilergebnis ihrer Studie. Bald: Kino, S. 68. Die Autorin verweist hier auch auf den Beitrag von Bausinger: Kultur ist mehr, S. 18 . 
kann der Besuchende Kinowerbung anschauen. Die Werbeumsätze werden nach Klickzahlen mit dem jeweiligen Kino abgerechnet. ${ }^{51}$

Die in diesem Beitrag dargestellten Eigenschaften und Funktionen von Kinos im ländlichen Raum können als ein erster Einblick in die Analyse des empirischen Materials betrachtet werden. Auf welche weiteren Strategien und Selbstverständnisse von Kinobetreibern und Publikum wir treffen, wird der Fortgang des Projektes zeigen. Im Jahr 2020 sollen drei weitere Kinoporträts aus dem Rheinland in Form von Kurzfilmen entstehen. Die Auswahl der Lichtspielhäuser wird dabei von ihrer Lage im Rheinland und ihrer Organisationsform abhängen.

\section{Linksammlung}

Alle Zugriffe am 30.3.2020.

https://alltagskulturen.Ivr.de/

https://heimatkino.de

https://heimatkino.de/kinokarte

https://heimatkino.de/episoden

https://hilfdeinemkino.de/\#kino

https://rheinische-landeskunde.lvr.de/de/alltagskultur/alltagskultur_filme/alltagskultur_filme_info.html

51 Weischer.Cinema GmbH \& Co. KG: Werbung kann Kinos retten, URL: https://hilfdeinemkino.de/\#kino. Kurz vor Redaktionsschluss, Anfang August 2020, lässt sich festhalten, dass das Corso Film Casino in Kaldenkirchen am 30.5.2020 seinen Spielbetrieb wieder aufgenommen hat, während das Kur-Theater Hennef die Wiedereröffnung für Ende August 2020 ankündigt.
Filme

Kurzfilme in der Reihe „Kinokultur im ländlichen Raum“:

Benda Film im Auftrag des LVR: Corso Film Casino Kaldenkirchen, Köln/Bonn 2019; URL: https://www.youtube.com/watch?v=TV1pd-2tr_w\&feature=youtube.

Benda Film im Auftrag des LVR: Kur-Theater Hennef, Köln/Bonn 2019; URL: https://www.youtube.com/ watch?v=er81gdblupg.

\section{Interviews}

Interview mit Helmut Töpfer am 21.2.2019 in

Kaldenkirchen.

Interviews mit Ingo Teusch, Petra Stratmann und Daniel Huys am 26.10.2019 in Hennef.

\section{Literatur und Quellen}

Augenblick. Konstanzer Hefte zur Medienwissenschaft 56/57 (2013): Erfahrungsraum Kino.

FFA Filmförderungsanstalt: Zukunftsprogramm Kino; URL: https://www.ffa.de/zukunftsprogramm-kino.html.

FFA-Presse- und Öffentlichkeitsarbeit: Corona-Krise: FFA-Präsidium beschließt Maßnahmenpaket für die deutsche Film- und Kinowirtschaft, 19.03.2020; URL: https:// www. ffa. de/aid=1394. html? newsdetail=20200319-1351_ corona-krise-ffa-praesidium-beschliesst-massnahmenpaket-fuer-die-deutsche-film-und-kinowirtschaft.

LVR Pressemeldungen: Kinokultur im ländlichen Raum, 15. Oktober 2019; URL: https://www.lvr.de/de/nav_main/ derlvr/presse_1/pressemeldungen/press_report_209217. jsp.

ntv, Politik: Hilfsprogramm für neue Technik. Bund investiert Millionensumme in Kinos; URL: https://www.n-tv.de/ politik/Bund-investiert-Millionensumme-in-Kinos-article21628701.html.

Presse- und Informationsamt der Bundesregierung (BPA): Pressemitteilung 227, 1.7.2019: 
Soforthilfeprogramm für Kinos auf dem Land startet Grütters: „Kulturort Kino auch abseits der Metropolen erhalten"; URL: https://www.bundesregierung.de/breg-de/ suche/soforthilfeprogramm-fuer-kinos-auf-dem-landstartet-gruetters-kulturort-kino-auch-abseits-der-metropolen-erhalten-1642378.

Katja Bald: Kino auf dem Land. Förderung der Filmkultur in strukturschwachen Regionen, in: Zeitschrift für Medienpädagogik 10 (2019); URL: https://journals.qucosa.de/ zfm/article/view/2019-10-bald/5.

Hermann Bausinger: Kultur ist mehr..., in: Eckart Frahm/ Holger Magel/Klaus Schüttler (Hg.): Kultur - ein Entwicklungsfaktor für den ländlichen Raum. Anregungen, Tips und Beispiele aus der Praxis, München 1994, S. 13-22.

\section{Winfried Gebhardt/Ronald Hitzler/Michaela Pfaden-} hauer (Hg.): Events. Soziologie des Außergewöhnlichen, Opladen 2000.

Stefan Gohlisch: Grütters: „Kinos sind geistige Tankstellen", in: Neue Presse, Kultur/Interview, 31.10.2019; URL: https://www.neuepresse.de/Nachrichten/Kultur/ Monika-Gruetters-im-Interview-Kinos-sind-geistige-Tankstellen.

Susanne Haake: „Ins Kino gehen war für mich die Krönung!" Narrative Modi der Erinnerung an das Kino, in: Augenblick. Konstanzer Hefte zur Medienwissenschaft 61 (2015), S. 63-81.

Franziska Heller: Erfahrungsraum Kino im Zeitalter der digitalen Reproduzierbarkeit, in: Augenblick. Konstanzer Hefte zur Medienwissenschaft 56/57 (2013), S. 187-202.

Andreas Hepp/Waldemar Vogelsang: Kino als MedienEvent. Dargestellt am Beispiel des Films „Titanic", in: Winfried Gebhardt/Ronald Hitzler/Michaela Pfadenhauer (Hg.): Events. Soziologie des Außergewöhnlichen, Opladen 2000, S. 239-262.

Marc Mensch: Zukunftsprogramm Kino: Das Bild schärft sich, 14.11.2019, für Blickpunkt:Film GmbH; URL: http://beta.blickpunktfilm.de/details/445523.

Florian Mundhenke: Von den Grenzen der Kinoerfahrung. Anmerkungen zum Kino als Erfahrungsraum im Zeitalter von Nutzungs- und Wahrnehmungsumbrüchen, in:
Augenblick. Konstanzer Hefte zur Medienwissenschaft 56/57 (2013), S. 203-220.

Florian Mundhenke: Postmoderne Cinéphilie. Die Veränderungen des sozialen Raums Kino als Reflexionen von Filmerfahrung in der digitalen Ära, in: Augenblick. Konstanzer Hefte zur Medienwissenschaft 64 (2015), S. 86-99.

Elizabeth Prommer: Film und Kino. Die Faszination der laufenden Bilder, Wiesbaden 2016.

Gerhard Schulze: Erlebnisgesellschaft. Kultursoziologie der Gegenwart, 2. Auflage, Frankfurt (Main) 2005. 
A.G./AG

$\mathrm{AHL}$

Anm.

Art.

BArch

BayHStA

Bd.

Best.

$\mathrm{Bl}$.

BLHA

BPA

bzW.

CDU

CFC

Co.

DCP

DDR

DEFA

Dir.

DJ

DNN

Dr.

DVD

EFKA

e.V.

FDGB

FDP

FF

$\mathrm{GmbH}$

GP

$\mathrm{H}$.

$\mathrm{Hg}$.

$\mathrm{HO}$

HStA Dresden

ISGV

$\mathrm{Jg}$.

KPD

$\mathrm{LMZH}$

LRA

LVR-ILR

MA GAP

MK

$\mathrm{mm}$

MNN

$\mathrm{Nr}$.

NS

NSDAP

ntv
Paragraf

Pfennig(e)

und

dreidimensional

Abbildung

Allgemeine Elektricitäts-Gesellschaft

Aktiengesellschaft

Archiv Hansestadt Lübeck

Anmerkung

Artikel

Bundesarchiv

Bayerisches Hauptstaatsarchiv München

Band

Bestand

Blatt

Brandenburgisches Landeshauptarchiv

Presse- und Informationsamt der Bundesregierung

beziehungsweise

Christlich-Demokratische Union

Corso Film Casino

Compagnie

Digital Cinema Packages

Deutsche Demokratische Republik

Deutsche Film AG

Direktor

Discjockey

Dresdner Neueste Nachrichten

Doktor

Digital Video Disc/Digital Versatile Disc

Frankfurter Kammerlichtspiele

eingetragener Verein

Freier Deutscher Gewerkschaftsbund

Freie Demokratische Partei

Filmförderungsanstalt

Gemeinschaft mit beschränkter Haftung

Gewerbepolizei

Heft

Herausgeber

Handelsorganisation

Sächsisches Staatsarchiv - Hauptstaatsarchiv Dresden

Institut für Sächsische Geschichte und Volkskunde Dresden

Jahrgang

Kommunistische Partei Deutschlands

Landesmedienzentrum Hamburg

Landratsamt

Landschaftsverband Rheinland-Institut für Landeskunde und Regionalgeschichte Marktarchiv Garmisch-Partenkirchen

Kultusministerium

Millimeter

Münchner Neueste Nachrichten

Nummer

Nationalsozialistisch, auch: Nationalsozialismus

Nationalsozialistische Arbeiterpartei Deutschlands

Fernsehnachrichtensender 


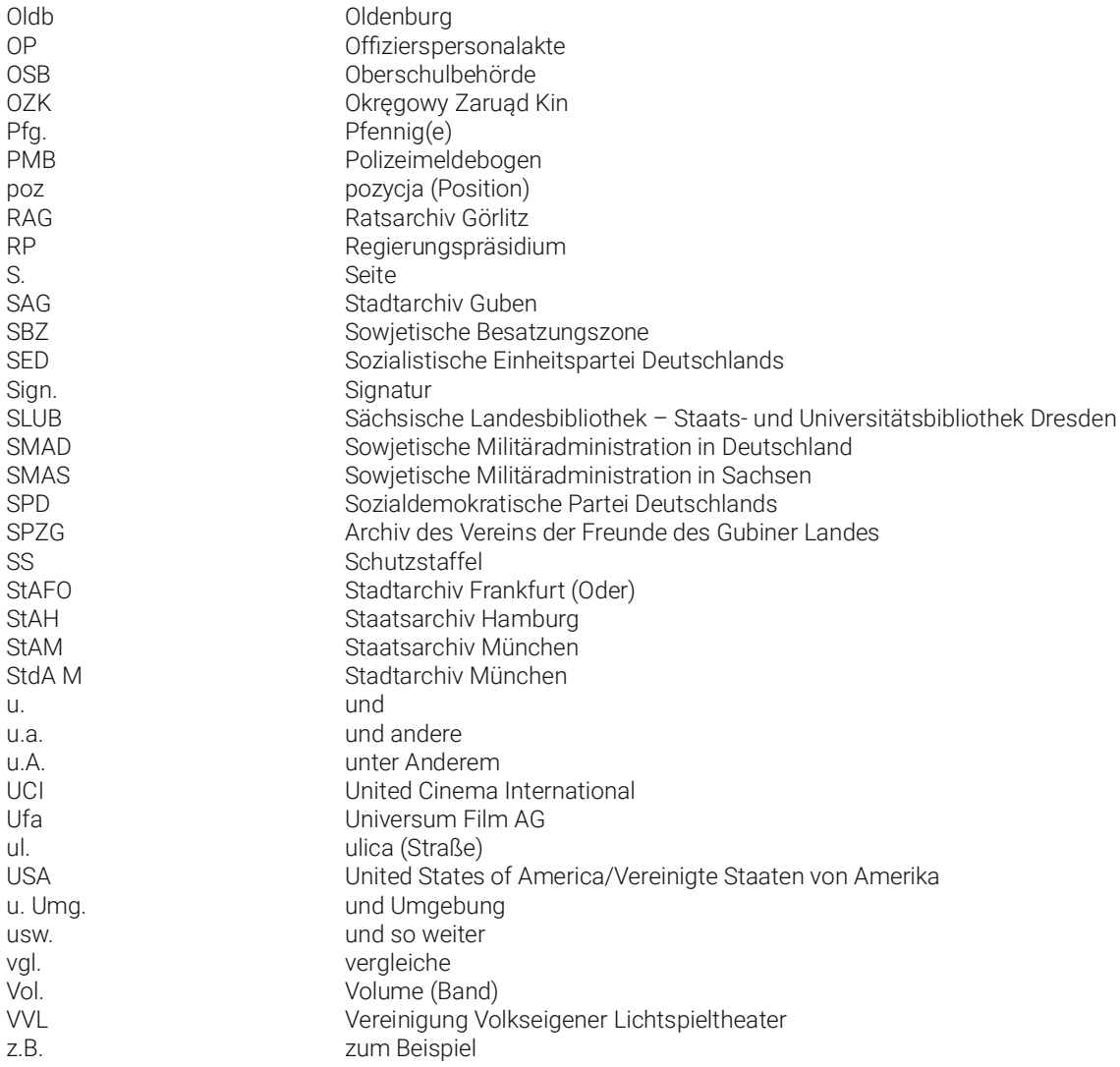

